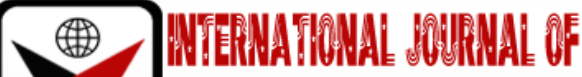

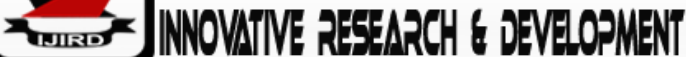

ISSN 2278-0211 (Online)

\section{Analysis of Stochastic Birth - Death Process with Mean, Variance and Matrix Models}

\author{
Agba M. E. \\ Lecturer, Department of Mathematics, Federal College of Education, Obudu, Nigeria \\ Egwe M. E. \\ Senior Lecturer, Department of Mathematics, University of Ibadan, Nigeria
}

\begin{abstract}
:
The mean and variance were generated from the probability generating function. The generator matrix A was gotten from the stochastic Birth-Death process. Applications for the study such as Data communication, Post office, Park place, Super markets, Traffic lights, Production system were highlighter as the importance of stochastic processes. Basic notations and definitions were given to understand the various concepts.
\end{abstract}

Keywords: Stochastic process, birth-death, mean, variance, matrix model and probability generating function

\section{Introduction}

The word stochastic is an adjective in English that describes something that is randomly determined and described mathematical objects called stochastic processes. Stirzaker (2005, pp. 45-48); Fugo (2011) enumerated areas of stochastic process such as Biology, Chemistry, Physics, Statistics, Technology, Engineering and Finance.

Yin and Zhang (2013, pp. 31-49) described Markov processes as states space with the population size, $X=$ $\left\{x_{t}, t \geq 0\right\}$, taking values in the set; $\mathbf{S}=\{0\} \cup \mathbb{N}$ is a discrete or continuous time, where $\mathbb{N}=\{1,2,3, \ldots, \mathbb{K}\}, \mathbb{K}$ denotes the smallest value.

Feller (1968); Yule (1998) explained birth-death rate as models for queuing process. Agba and Ugbebor (2017, pp. 1-5) derived mathematical formula for the Extinction Probability with a condition when the birth rate is greater than the death rate. Okoro (2013, pp. 23-28) worked on markovianqueuing model as birth-death process in Epidemiology analysis. Walsh (2006) studied Stochastic processes in relation to population growth and considered birth and deathprocess represented as $\lambda$ and $\mu$ respectively, and birth-death brings about increase or decrease in population. $\underline{\text { Chiyo }}$ (2018) found that ذiroemonKimuro from Japan died at the age of one hundred and twenty-two years, one hundred and sixty-four days (122 years, 64 days), the oldest known living person is Kane Tanaka of Japan, age one hundred and seventy-seven years, thirty-three days (177 years, 33 days). Stochastic Birth-Death equations were used to generates infinitesimal generator matrix $\mathrm{A}$ and the probability generating function was used to derived the mean and variance respectively.

\section{Derivation of the Mean and Variance for Birth - Death Processes}

We used the method of moments to find the mean and variance from the generating function. Differentiating once and then twice we have the mean and variance respectively as follows:

$$
G(t, Z)=\sum_{n=0}^{\infty} p_{n}(t) z^{n}
$$

$\frac{\partial G}{\partial z}(t, z)=\sum_{n=0}^{\infty} n p_{n}(t) z^{n-1}$

$$
\frac{\partial G}{\partial z}(t, 1)=\sum_{n=0}^{\infty} p_{n}(t)
$$

and

$\frac{\partial^{2} G}{\partial Z}(t, z)=\sum_{n=0}^{\infty}(n-1) n p_{n}(t) z^{n-2}$
$\quad=\sum_{n=0}^{\infty}\left(n^{2}-n\right) p_{n}(t) z^{n-2}$ 
$\frac{\partial^{2} G}{\partial Z}(t, 1)=\sum_{n=0}^{\infty}\left(n^{2}-n\right) p_{n}(t)$

$=\sum_{n=0}^{\infty} n\left(n-1 p_{n}(t)(2)\right.$

Let $\mathrm{n}(t)$ denotes the mean at time $t$ and $\partial^{2}(t)$ the variance at time $t$. Differentiating (1) once and then twice, thensetting $z=1$, after each differentiation. The variance is the second moment of probability distribution about the mean.

$$
\partial^{2}(t)=E[n(n-1)]+\bar{n}(\mathrm{t})-\bar{n}^{2}(\mathrm{t})
$$

\subsection{Derivation of the Mean}

Given the generating function:

$G(t, z)=\frac{\mu(1-z)-(\mu-\lambda) e^{-(\lambda-\mu) t}}{\lambda(1-z)(\mu-\lambda z) e^{-(\lambda-\mu) t}}$

$=\frac{\left(-\mu+\lambda e^{-(\lambda-\mu) t}\right)-\left(\lambda-\lambda z-(\mu-\lambda z) e^{-(\lambda-\mu) t)}-\left[\mu(1-z)-(\mu-\lambda z) e^{-(\lambda-\mu) t}\right]\left[-\lambda+\lambda e^{-(\lambda-\mu) t]}\right.\right.}{\left[\lambda-\lambda z-(\mu-\lambda z) e^{-(\lambda-\mu) t}\right]^{2}}$

$=\frac{\left(-\mu+\lambda e^{-(\lambda-\mu) t}\right)\left(-(\mu-\lambda) e^{(\lambda-\mu) t}\right)-\left(-(\mu-\lambda) e^{-(\lambda-\mu) t}\right)\left(-\mu+\lambda e^{-(\lambda-\mu) t}\right)}{\left[-(\mu-\lambda) e^{-(\lambda-\mu) t}\right]^{2}}$

$=\frac{\left.\left(-\mu+\lambda e^{-(\lambda-\mu) t}\right)(\lambda-\mu) e^{-(\lambda-\mu) t}-(\lambda-\mu) e^{-(\lambda-\mu) t}\right)\left(-\lambda+\lambda e^{-(\lambda-\mu) t}\right)}{\left[-(\mu-\lambda) e^{-(\lambda-\mu) t}\right]^{2}}$

$=\frac{-\mu \lambda e^{-(\lambda-\mu) t}-\lambda-\lambda e^{-(\lambda-\mu) t}}{(\mu-\lambda) e^{-(\lambda-\mu) t}}$

$=\frac{-\mu+\lambda}{B}$

For, $B=(\lambda-\mu) e^{-(\lambda-\mu) t}$

$=\frac{\lambda-\mu}{(\lambda-\mu) e^{-(\lambda-\mu) t}}$

$=\frac{1}{e^{-(\lambda-\mu) t}}$

$\frac{\partial G}{\partial Z}(1, t)=e^{-(\lambda-\mu) t}=\bar{n}(\mathrm{t})$

Hence, $\frac{\partial G}{\partial Z}(1, t)=e^{-(\lambda-\mu) t}=E(n)=\bar{n}(\mathrm{t})$

For $\lambda=\mu$, we have the mean from (6) as;

$$
E(n)=\bar{n}(t)=n_{0}
$$

\subsection{Derivation ofthe Variance} produced.

We shall proceed to derive the variance of the second moment of the expected number of species or individuals

By definition of variance:

$V(N)=E\left(N^{2}\right)-[\mathrm{E}(N)]^{2}$

$$
E\left(N^{2}\right)=\sum_{n=0}^{\infty} n^{2} p_{n} E(N)
$$

But, $N^{2}=\mathrm{N}(\mathrm{N}-1)+\mathrm{E}(\mathrm{N}$

$$
\begin{aligned}
& \Rightarrow E\left(N^{2}\right)=E[N(N-1)]+E(N) \\
& E\left[N(N-1)=\sum_{n=0}^{\infty} n(n-1) p_{n}(t)\right.
\end{aligned}
$$

$\sum_{n=0}^{\infty} n(n-1) p_{n}(\mathrm{t})=-(\lambda+\mu) \sum(n-1) n^{3} p_{n}(t)+\mu \sum(n+1)(n-1) n^{2} p_{n+1}(t)$

$$
+\lambda \sum(n-1)^{2} n^{2} p_{n-1}(\mathrm{t})
$$

$=n-1\left[-(\lambda+\mu) \sum n^{3} p_{n}(\mathrm{t})+\mu \sum(n+1) n^{2} p_{n+1}(t)+\sum(n-1) n^{2} p_{n-1}(t)\right]$

Substitute,

$(n+1) n=(n+1)^{2}-(n+1)$

$(n-1) n=(n-1)^{2}-(n-1)$

Then,

$$
n_{2}{ }^{1}=(n-1)\left[-(\lambda+\mu) \sum n^{3} p_{n}(\mathrm{t})+\lambda \sum(n-1)^{2} n^{2} p_{n-1}(\mathrm{t})+\right.
$$

$\left.\lambda \sum n(n-1) p_{n-1}(t)+\mu \sum(n+1)^{2} n p_{n+1}(\mathrm{t})-\mu \sum(n-1) p_{n+1} \mathrm{t}\right]$ 
Let, $\mathrm{n}-1=\mathrm{n}$, and $\mathrm{n}+1=\mathrm{n}$, we have

$$
n_{2}{ }^{1}=-(\lambda+\mu) \sum n^{3} p_{n}(\mathrm{t})+\lambda \sum n^{3} p_{n}(\mathrm{t})+\lambda \sum n^{2} p_{n}(t)
$$

$+\mu \sum n^{3} n p_{n+1}(\mathrm{t})-\mu \sum n^{2} p_{n}(\mathrm{t})$

$=\lambda \sum n^{2} p_{n}(\mathrm{t})-\mu \sum n^{2} p_{n}(\mathrm{t})$

$=(\lambda-\mu) \sum n^{2} p_{n}(\mathrm{t})$

$=(\lambda-\mu) e^{(\lambda-\mu) t}$

$$
\begin{aligned}
& =\frac{\lambda+\mu e^{(\lambda-\mu) t}}{(\lambda-\mu)}\left[e^{(\lambda-\mu) t}-\left(e^{t(\lambda-\mu)^{2}}\right]\right. \\
& =\frac{\left(n_{0}\right) \lambda-\mu e^{(\lambda-\mu) t}}{(\lambda-\mu)} e^{(\lambda-\mu) t}\left[1-e^{(\lambda-\mu) t}\right] \\
& =\frac{n_{0}(\lambda+\mu) e^{2(\lambda-\mu) t}}{(\lambda-\mu) t}\left[1-e^{(\lambda-\mu) t}\right]
\end{aligned}
$$

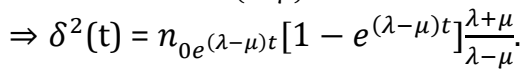

If $\lambda=\mu$, the variance in (7) is given as:

$\delta^{2}(\mathrm{t})=2 n_{o} \lambda t$.

\subsection{Matrix Analytic Method}

Applying the Markov property and the infinitesimal transitional probabilities, a continuous time analogue of the transition matrix can be defined.

$p_{n}(\mathrm{t}+\Delta t)=p_{n}(t) \lambda(n-1) \Delta t+p_{n+1}(\mathrm{t}) \mu(\mathrm{n}+1) \Delta t+p_{n}(t)(1-[\lambda(n)+\mu(n)] \Delta t)+o(\Delta t)$

Subtracting $p_{n}(t)$ then dividing by $\Delta t$, and letting $\Delta t \rightarrow 0$, leads to;

$\left.\frac{d p_{n}}{d t}=\lambda(\mathrm{n}-1) p_{n-1}(\mathrm{t})+\mu(\mathrm{n}+1) p_{n+1}(\mathrm{t})-(\lambda+\mu) n p_{n}(\mathrm{t})\right)(8)$

For, $n=0,1,2,3, \ldots \quad \mathbb{N}$

In matrix notation, it can be expressed as

$\frac{d p_{n}}{d t}=A p$

Where,

$P(t)=\left(p_{0}(t), \quad p_{1}(t) \ldots ., p_{N}(t)\right)^{T}$.

Computation of $A$ from (8)yields:Type equation here.

For,

$n=0: p_{0}=0, p_{1}=\mu(1)$,

$n=1: p_{0}=0, p_{1}=-\left[\lambda(1)+\mu(1), \quad p_{2}=\mu(2)\right.$

$n=2: \quad p_{0}=0, \quad p_{1}=\lambda(1), \quad p_{2}=-[\lambda(2)+\mu(2)$

$n=3: \quad p_{0}=0, \quad p_{1}=0, \quad p_{2}=2 \lambda, \quad p_{3}=-[\lambda(3)+3(\mu)]$,

$p_{4}=4 \mu$

$n=4: \quad p_{0}=0, \quad p_{1}=0, \quad p_{2}=0, \quad p_{3}=3 \lambda, \quad p_{4}=-[\lambda(4)+4(\mu)]$

$p_{5}=5 \mu$

Hence, the coefficients of $p_{0}, p_{1}, p_{2}, \ldots p_{N}$ are represented as follows:

$\mathrm{P}(\mathrm{t})=\left[\begin{array}{cccccc}0 & \mu(1) & \mu & \ldots & 0 & \\ 0 & -[\lambda(1)+\mu(1)] & \mu(2) & \ldots & & 0 \\ 0 & \lambda(1) & -[\lambda(2)+\mu(2)] & \ldots & \mu(2) & \\ \vdots & \vdots & \vdots & & & \vdots \\ 0 & 0 & 0 & & & \ldots-\mu(N)\end{array}\right]$

Matrix $A$ is denoted as:

$\mathrm{A}=\left[\begin{array}{cc}0 & 0 \\ 0 & -\lambda(1)-\mu(1) \\ 0 & \mu(2) \\ \vdots & \vdots \\ 0 & 0\end{array}\right.$

$$
\left.\begin{array}{cccc}
0 & \ldots & 0 & \\
\lambda(1) & \ldots & 0 & \\
-[\lambda(2)+\mu(2)] & \ldots & 0 \\
\vdots & \vdots & \vdots & \\
0 & & & \ldots-\mu(N)
\end{array}\right]
$$

Matrix $A$ is referred to as the infinitesimal generator matrix or simply the generator matrix.

The differential equation:

$\frac{d p}{d t}=A p$

Is known as the backwardKolmogorov differential equations,where

$P=p_{n}(\mathrm{t})$, is the matrix infinitesimal transition probabilities and

$P(t)=\left[p_{0}(\mathrm{t}), p_{1}(\mathrm{t}), \ldots p_{N}(\mathrm{t})\right]$ is a row vector representing the probabilitydistribution and

$\sum_{n=0}^{\infty} p_{n}(\mathrm{t})=1$

The generator matrix $A$ has a zero eigenvalue with corresponding eigenvector

$\left(\begin{array}{llll}1 & 0 & 0 & \ldots\end{array}\right)^{T} . \quad$ The other eigenvalues are negative or have negative real part.

Where the first rows and first columnsare not deleted, thedeterminant is zero (singular matrix) but where they are deleted, A is non - singular.

Using $|\operatorname{det}| A-I_{n} t \mid$, we have; 
$\operatorname{det}\left|A-I_{n} t\right|=\left(\begin{array}{ccc}-\lambda(1)-\mu(1)-x & \mu(1) & 0 \\ \mu(2) & -\lambda(2)-\mu(2)-x & \lambda(2) \\ 0 & \mu(3) & -\lambda(3)-\mu(3)-x\end{array}\right)$

Suppose $h_{n}$ is the determinant of the above matrixanddisregard the coefficients

of $\lambda, \mu$, and $\lambda+\mu$.

$\left.\operatorname{det}\left|A-I_{n} t\right|=-(\lambda+\mu+t)(-\lambda-\mu-t)-\lambda \mu\right)-\lambda[\mu(-\lambda-\mu-t)]$

Substitute,

$f=(-\lambda-\mu-t)=-(\lambda+\mu+t)$

And

$g=-\lambda \mu$

$\operatorname{det}\left|A-I_{n} t\right|=\mathrm{f}\left(f^{2}-\lambda \mu\right)-\lambda \mu f$

$=f\left(f^{2}+\mathrm{g}\right)+f g$

$f=0$, and $f+g=0$

$h_{n}$ is thedeterminant of $A$ and;

$h_{1}=f+g=-(\lambda+\mu+t)-\lambda \mu$

But,

$x_{n}=\operatorname{det}\left|A-I_{n} t\right|$

$=\operatorname{det}|A|$

From (10)

$x_{n}=f+g+g$

$=(f+g) h_{n-1}+g h_{n-2}$

If,$n=2 k$, then,

$h_{n+1}=\left(f^{n}+(n-1)\right) f^{n-2}+a_{1}{ }^{n+1} f^{n-4} g^{2}+a_{2}{ }^{n+2} f^{n-6} g^{3}+\ldots+a_{k-2}{ }^{n+1} f^{2} g^{k-1}$

$\left.+g^{k}\right) h_{1}+\left(f^{n-1} g+\left(\begin{array}{c}n+1 \\ 0\end{array}\right)(n+3) f^{n-3} g^{2}+\left(\begin{array}{c}n+1 \\ 1\end{array}\right) f^{n-5} g^{3}+\right.$

$+\ldots, \quad+\left(\begin{array}{c}n+1 \\ k-2\end{array}\right) f g^{k}$.

By induction on $n$;

$a_{1}{ }^{n+1}=(n-3)(k-1), \quad($ for,$n=2 k)$

$\begin{aligned} & \left.=2 k^{2}-5 z+3\right) . \\ a_{2}{ }^{n+2} & =\sum_{z=2}^{k-1}\left(2 z^{2}-5 z+3\right)+\sum_{z=3}^{k-1}\left(2 z^{2}-7 z+6\right)\end{aligned}$

Also

$h_{n}=\left(f^{n-1}+(n-2) f^{n-3} g+a_{1} f^{n-5} g^{2}+a_{2}{ }^{n} f^{n-7} g^{3}+\ldots+a_{k-2}{ }^{n} f^{2} g^{k-1}+g^{k}\right) h_{1}$

$+\left(f^{n-2} g+(n-3) f^{n-4} g^{2}+\left(\begin{array}{c}n \\ 1\end{array}\right) f^{n-6} g^{3}+\ldots,+\left(\begin{array}{c}n \\ k-3\end{array}\right) f^{2} g^{k-1}+g^{k}\right)$

$a_{2}{ }^{n}=\sum_{z=2}^{k-2}\left(2 z^{2}-5 z+3\right)+\sum_{z=3}^{k-1}\left(2 z^{2}-7+6\right)$

$\left(\begin{array}{l}n \\ 1\end{array}\right)=\left(\begin{array}{l}n \\ 2\end{array}\right)=a_{1}{ }^{n+1}$

$\left(\begin{array}{l}n \\ 1\end{array}\right)=a_{1}^{n-1}$

$\left(\begin{array}{l}n \\ 1\end{array}\right)=a_{1}^{n}=0$

\section{Conclusion}

We derived the mean and variance from the probability generating function (4) and computed values for $n=0,1,2, \ldots$, from the Birth-Death process. From our computerization, the first rows and first columns were deleted in order to have a unique solution not singular matrix with zero determinant. Arbitrary value $h_{n}$ was assigned as the $\operatorname{det}\left|A-I_{n} t\right|$ for the objective to be achieved. The importance of the mean derivation enable us to ascertain the number of peoples infected with diseases like Typhoid, Small pox, Malaria and Covid-19 (Coronavirus Disease 2019) as one of the deadly diseases in the community, society and the world at large. W.H.O, (2021) said to overcome the menace, the government, individuals and non-governmental organizations have to profile solutions to eradicate or eliminate the diseases, a good example is the Covid-19 vaccine (Astrazeneca),manufacturedin India and distributed to assist other countries.

\section{References}

i. Agba, M.E. \&Ugbebor, O. O. (2017). Stochastic Differential Equation on Extinction Probability. Intl J of Sci and Res. (IJSR). ISBN: 2319-7064.

ii. Allen, L.J. (2003). An Introduction toStochastic Process with Application to Biology.Prentice Hall, Upper Saddle River. 
iii. Chiyo, M. (2018). Guinness World Records, ®,(GWR).

iv. Feller, W. (1968). An Introduction to Probability Theory and Its Application.Wiley, New York.

v. Fugo, T. (2011). Birth-Death models.J. Appl. Prob. 6, 205-210.

vi. Okoro, O. J. (2013). OnMarkovian Queueing Model as Birth-Death Process. Global Journal of Sciences Frontier Research. ISSN: 2249-4926.

vii. Stirzaker, D. (2005). Stochastic Processes and Models. Oxford University Press, p. 45, ISBN: 878-0-19-856814-8.

viii. Walsh, S.(2006). Extinction. http://nitro.biosci.arizona.edu/zdownload/talks/2006/Extinction.(Retrieved September 5, 2008).

ix. W.H.O. (2021). World Health Organization: Covid-19 Strategy. Retrieved May, 2021, from http://www.who.int//covid-19strategy.

x. Yin, G. G. \&Zhang, Q.(2013). Stochastic Modeling and Applied Probability. Continuous-Time Markovchains and Applications. Springer, New York. Yule, G. (1998). A Mathematical Theory ofEvolution. The Royal Society of London, 213, 21-87. 